\title{
Diagnostic importance of faecal markers in long-term monitoring of anti-TNF- $\alpha$ therapy in primary responders with Crohn's disease
}

\author{
Liliana Łykowska-Szuber ${ }^{1}$, Katarzyna Klimczak ${ }^{1}$, Piotr Eder ${ }^{1}$, Iwona Krela-Kaźmierczak ${ }^{1}$, Kamila Stawczyk-Eder ${ }^{1}$, \\ Michał Michalak², Adam Studniarek³ ${ }^{3}$ Tomasz Kościński ${ }^{3}$, Aleksandra Szymczak ${ }^{1}$, Krzysztof Linke ${ }^{1}$ \\ ${ }^{1}$ Department of Gastroenterology, Human Nutrition and Internal Diseases, Poznan University of Medical Sciences, Poznan, \\ Poland \\ 2Department of Computer Science and Statistics, Poznan University of Medical Sciences, Poznan, Poland \\ ${ }^{3}$ Department of General Surgery, Gastroenterological and Endocrinological Surgery, Poznan University of Medical Sciences, \\ Poznan, Poland
}

Key words: anti-TNF antibodies, faecal markers, Crohn's disease.

Address for correspondence: Piotr Eder MD, PhD, Department of Gastroenterology, Human Nutrition and Internal Diseases, Poznan University of Medical Sciences, 49 Przybyszewskiego St, 60-355 Poznan, Poland, phone: +48 698 050 797, e-mail: piotr.eder@op.pl

\begin{abstract}
Introduction: Monitoring the response to biological treatment in Crohn's disease (CD) is a very important element of the therapeutic optimisation.

Aim: To evaluate the usefulness of measuring calprotectin, lactoferrin, and myeloperoxidase in stool as markers of long-term clinical and endoscopic response to anti-tumour necrosis factor $\alpha$ (anti-TNF) treatment in CD.

Material and methods: The studied group consisted of 35 CD patients treated with anti-TNF- $\alpha$ antibodies. Clinical activity was evaluated using Crohn's Disease Activity Index (CDAI), and the exacerbation of endoscopic changes was evaluated using a Simple Endoscopic Score for Crohn's Disease (SES-CD). The concentration of calprotectin, lactoferrin, and myeloperoxidase was measured using the ELISA method. All measurements were performed three times - before, after 3 months, and after a year of therapy.

Results: During anti-TNF treatment the concentrations of all measured faecal markers decreased significantly in relation to baseline values. We observed a significant correlation at all time-points: before the therapy, after 3 months, and 12 months after starting the therapy, between the concentration of calprotectin and SES-CD, calprotectin and CDAl, as well as between lactoferrin and SES-CD, and lactoferrin and CDAI. Myeloperoxidase correlated with both SES-CD and CDAI only after 1 year of treatment.

Conclusions: Faecal calprotectin and lactoferrin are valuable markers of clinical and endoscopic activity of CD in patients treated with anti-TNF antibodies. They are useful in monitoring the response to treatment. The usefulness of myeloperoxidase in this respect remains controversial.
\end{abstract}

\section{Introduction}

Crohn's disease (CD) is an inflammatory bowel disease (IBD). The inflammatory changes in CD can be present in any part of the gastrointestinal tract. The disease presents as intestinal wall inflammatory infiltration, strictures of the intestinal lumen, and/or fistulas. It is a chronic disease with episodes of exacerbations and remissions. During the exacerbation period diarrhoea, bloody stools, and abdominal pain are the most common presenting symptoms. The clinical picture very often does not reflect the typical characteristics of the disease, which creates a significant diagnostic problem [1]. Crohn's Disease Activity Index (CDAI) is the most commonly used indicator of disease exacerbation in clinical studies and in every day practice [2]. It includes a number of criteria such as: present state of being, abdominal pain, number of bowel movements, haematocrit value, body weight, and administered drugs. Numerous studies have proven that the clinical picture does not always correlate with the endoscopic activity index [2-5]. Mucosal healing $(\mathrm{MH})$ is the most 
important component of CD remission. The main goal of treatment in CD focuses on obtaining endoscopic remission. The $\mathrm{MH}$ is the criterion of the treatment's effectiveness [6]. Doses and time of drug administration should be adjusted to the patient's individual needs. It is only possible through a precise evaluation of the treatment's effectiveness. As mentioned above, the necessary element is the evaluation of endoscopic changes. Mucosal healing is a necessary criterion in order to obtain permanent remission of the disease. The gold standard to evaluate this criterion is endoscopy. However, it is an expensive, often scarcely available method that requires a burdensome test preparation. This is the reason why we search for a cheap and easily accessible test with high sensitivity that will reflect the grade of intestinal wall inflammatory infiltration and therefore will be a good early diagnostic tool for the assessment of treatment effectiveness and detection of early exacerbations of IBD. In the literature it has been stated that inflammatory biomarkers in stool can meet these expectations $[7,8]$. The most commonly investigated markers are calprotectin, lactoferrin, and to a lesser extent myeloperoxidase. Numerous studies have proven that their increased concentration correlates with the degree of inflammatory changes in the gastrointestinal tract. A lot of researchers conclude that the concentration of these biomarkers can indirectly reflect the endoscopic changes in the intestines [9-11].

Biological treatment of IBD has been a significant accomplishment in this field of medicine in the last decade. These drugs have been the most effective in the treatment of CD up to this date. They are capable of inducting permanent and full remission of the disease, which mostly depends on the grade of intestinal $\mathrm{MH}$. During biological treatment, it is extremely important to evaluate the response to treatment. Remission of endoscopic changes is the basic criterion to end the treatment [12]. The number of research studies that assess the usefulness of these faecal markers in monitoring the response to treatment of anti-tumour necrosis factor $\alpha$ (anti-TNF) drugs is still very low.

Calprotectin is a protein with molecular weight of $36 \mathrm{kDa}$ that belongs to the S100 low-molecular proteins family. It is a protein that expresses the ability to bind calcium and zinc ions, which determines its bacteriostatic function. It is mostly produced by neutrophils making up 30-60\% of cytological proteins, and to a lesser extent by monocytes and reactive macrophages. Calprotectin is released into the extracellular space when the neutrophils and/or monocytes are stimulated by pro-inflammatory cytokines $[13,14]$. Lactoferrin is a glycoprotein from the transferrin family, with a molecular mass of approximately $80 \mathrm{kDa}$. It is charac- terised by the ability to bind iron ions. It is produced by secretory epithelial cells and is present in breast milk, saliva, cerebrospinal fluid, and seminal fluid. It is also present in secondary and tertiary granulation, during which lactoferrin is released directly into the bloodstream or inflammatory areas [15].

Myeloperoxidase is an enzyme that belongs to the peroxidases group. It is a catalyst in the chemical reaction forming hypochlorous acid $(\mathrm{HOCl})$, which is a chemical compound with strong bactericidal and antiviral properties. Myeloperoxidase is present in the granularity of neutrophils and is released during inflammatory reactions [16].

\section{Aim}

The goal of our research study is to evaluate the usefulness of the measurement of calprotectin, lactoferrin, and myeloperoxidase concentrations in stool in monitoring long-term clinical response and endoscopic changes to anti-TNF- $\alpha$ treatment in CD patients who initially responded well to the treatment.

\section{Material and methods}

Patients with CD who were treated with antiTNF- $\alpha$ were qualified as part of the therapeutic National Health Care program in the Department of Gastroenterology and Internal Medicine in Medical University of Poznan during the years 2009-2013. The diagnosis was made based on the patient's history and laboratory, endoscopic, and histopathological results. Exclusion criteria consisted of: pregnancy, fistulas, present stoma, intestinal resections, and administration of non-steroidal anti-inflammatory drugs at least 3 months before qualification for the study. Patients who did not respond to induction treatment were not qualified for the continuation of biological treatment and therefore were excluded from the study. A good response to treatment was defined as a decrease in CDAI index by a minimum of 70 points, and as a clinical remission CDAI $<150$ points. During the study patients did not receive any antibiotics; the disease remission was free from corticosteroids.

Clinical activity was evaluated using the CDAI [2]. Every patient underwent a colonoscopy with terminal ileum intubation performed by an experienced endoscopic specialist. Endoscopic activity was evaluated using the Simple Endoscopic Score for Crohn's Disease (SES-CD) [4]. Before the colonoscopy we obtained a stool sample in order to measure the concentration of calprotectin, lactoferrin, and myeloperoxidase. This sample was frozen and stored at $-20^{\circ} \mathrm{C}$ until the markings were performed. After defrosting to room temperature, we measured the concentration of the specific faecal 
markers. Calprotectin was measured using the PhiCal ${ }^{\oplus}$ Calprotectin ELISA test (Immundiagnostik, Germany), lactoferrin using IBDScan ELISA test (TechLab, USA), and myeloperoxidase using ELISA test (Immundiagnostic, Germany). The evaluation of the above parameters (CDAI, SES-CD, faecal markers) was performed three times: before admission to the study, after the end of induction treatment (after 3 months - at week 12), and after 12 months of therapy (at week 56). Patients were

Table I. Clinical characteristics of the study group $(n=35)$

\begin{tabular}{|c|c|c|}
\hline Feature & Number & Percentage \\
\hline Age, median (range) [years] & $34.5(18-53)$ & \\
\hline Gender (female/male) & $20 / 15$ & $57.1 / 42.8$ \\
\hline \multicolumn{3}{|l|}{ Disease location: } \\
\hline Colon & 8 & 22.8 \\
\hline Ileocolon & 27 & 77.2 \\
\hline Duration of the disease [years] & 4.5 & \\
\hline Smoking: no/yes & $32 / 3$ & $91.4 / 8.6$ \\
\hline \multicolumn{3}{|l|}{ Maintenance treatment: } \\
\hline 5-Aminosalicylic acid & 32 & 91.4 \\
\hline Thiopurine & 28 & 80 \\
\hline \multicolumn{3}{|l|}{ Metronidazole: } \\
\hline Week 0 & 3 & 8.6 \\
\hline Week 12 & 0 & \\
\hline Week 56 & 0 & \\
\hline \multicolumn{3}{|l|}{ Corticosteroids: } \\
\hline Week 0 & 14 & 40 \\
\hline Week 12 & 0 & \\
\hline Week 56 & 0 & \\
\hline Adalimumab & 14 & 40 \\
\hline Infliximab & 21 & 60 \\
\hline
\end{tabular}

Table II. Change in clinical activity assessed by Crohn's Disease Activity Index (CDAI) and endoscopic activity assessed by Simple Endoscopic Score for Crohn's Disease (SES-CD) after 12 weeks, and after 1-year (56 weeks) of anti-TNF therapy in the study group

\begin{tabular}{lcc} 
Week & CDAI & SES CD \\
\hline 0 & $283($ IQR: 199-334) & 13.5 (IQR: 7-20) \\
\hline 12 & $80($ IQR: 54-145) & $6($ IQR: 4-8) \\
\hline 56 & $80($ IQR: $55-150)$ & $6($ IQR: 4-14)
\end{tabular}

treated as part of the National Health Care program (NFZ) using infliximab and adalimumab. Infliximab was given in dosages of $5 \mathrm{mg} / \mathrm{kg}$ of body weight at weeks 0,2 , and 6 and then every 8 weeks. Adalimumab was administered every 2 weeks. The initial dosage was $160 \mathrm{mg}$, the next was $80 \mathrm{mg}$, and the following was $40 \mathrm{mg}$.

The study was approved by the Bioethics Committee at Poznan University of Medical Sciences (Decision No 93/09).

\section{Statistical analysis}

The analysed data were not consistent with standard distribution (Shapiro-Wilk test). That is why the data were analysed using distribution free test. The results were presented as medians and interquartile ranges (IQR).

In order to measure the significant differences in the marker levels during treatment (three measurement points) the Friedman test was performed. In cases of significant differences post-hoc tests were performed to designate the homogenous groups. The correlations between the analysed parameters were measured using Spearman's rank correlation coefficient, and the statistical significance using Student's $t$-test. The statistical analysis was performed using Statistica 10.0 (StatSoft) software. All the tests were considered statistically significant when $p<0.05$.

\section{Results}

Initially 50 patients were qualified into this study. After 3 months of therapy the clinical response was obtained in 35 patients. This group was analysed during our study. The clinical characteristics of patients are presented in Table I. The change in CDAI and SES-CD after 12 weeks, and after 1-year of anti-TNF treatment in the study group is shown in Table II. The median calprotectin concentration in stool before the administration of biological treatment was 178.9 (IQR: 112.3-271.8) $\mathrm{mg} / \mathrm{l}$, lactoferrin 112 (IQR: 42.2-723.6) $\mu \mathrm{g} / \mathrm{ml}$, and myeloperoxidase 9136.5 (IQR: 4000-20956) ng/ml. After 3 months of treatment all of the above parameters decreased. In this group, the median concentration of calprotectin was 49.9 (IQR: 11.6-169.6) mg/l, lactoferrin 18.4 (IQR: $2.4-77) \mu \mathrm{g} / \mathrm{ml}$, and myeloperoxidase 5595.2 (IQR: 1358.6-11255.8) ng/ml. After a year of treatment median concentrations of the above markers were as follows: calprotectin 44.3 (IQR: $11.4-200) \mathrm{mg} / \mathrm{l}$, lactoferrin 15.8 (IQR: 3.5-96.2) $\mu \mathrm{g} / \mathrm{ml}$, and myeloperoxidase $5540.5 \mathrm{ng} / \mathrm{ml}$ (IQR: 1525.5-16010.3) ng/ml. Statistically significant differences were reported in regards to the concentrations of calprotectin (Figure 1), lactoferrin (Figure 2), and myeloperoxidase (Figure 3) between the groups before the treatment adminis- 


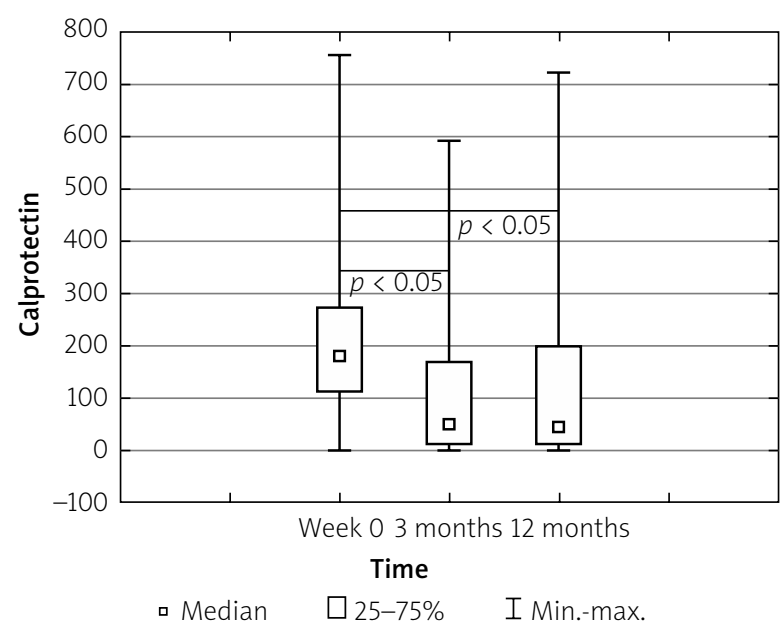

Figure 1. Median faecal calprotectin concentrations before anti-TNF treatment, after 3 months, and after 12 months of the therapy

tration, at the end of the induction period, and after a year of treatment. There were no statistically significant differences when comparing the concentration of assessed faecal markers at week 12 and after a year of treatment (Figure 1-3). After 56 weeks of the therapy in three patients there was an exacerbation of the disease. In these patients we observed increased concentrations of all faecal markers in comparison to the measures after induction of treatment (calprotectin: 195.4 vs. $439.3 \mathrm{mg} / \mathrm{l}, 31.2$ vs. $372.1 \mathrm{mg} / \mathrm{l}$, 62.86 vs. $722.61 \mathrm{mg} /$; lactoferrin: 60.1 vs. $363.2 \mu \mathrm{g} /$ $\mathrm{ml}, 1775.1$ vs. $3727.8 \mu \mathrm{g} / \mathrm{ml}, 4.56$ vs. $711.4 \mu \mathrm{g} / \mathrm{ml}$; myeloperoxidase: 725.4 vs. $18235.5 \mathrm{ng} / \mathrm{ml}, 1601.2$ vs. $2313.2 \mathrm{ng} / \mathrm{ml}, 4679.4$ vs. $26469.5 \mathrm{ng} / \mathrm{ml})$. The CDAl values in these patients during the flare of the disease were 360,480 , and 460 , respectively.

We obtained a correlation between the concentration of calprotectin and lactoferrin, and the endoscopic activity index SES-CD as well as CDAI both before the patients were qualified for treatment as well as 3 and 12 months after the treatment had been initiated (Tables III-V). The highest degree of correlation was observed between CDAI, SES-CD, and calprotectin after 12 months of treatment (Table $\mathrm{V}$ ). The concentration of myeloperoxidase correlated with the endoscopic activity index SES-CD after 3 months of therapy and with CDAI after 12 months of therapy (Tables III, V). There was no correlation between CDAI, SES-CD, and the concentration of myeloperoxidase in stool before the initiation of treatment (Table III). There was also no correlation between CDAI and the concentration of myeloperoxidase in stool after 3 months of therapy (Table IV).

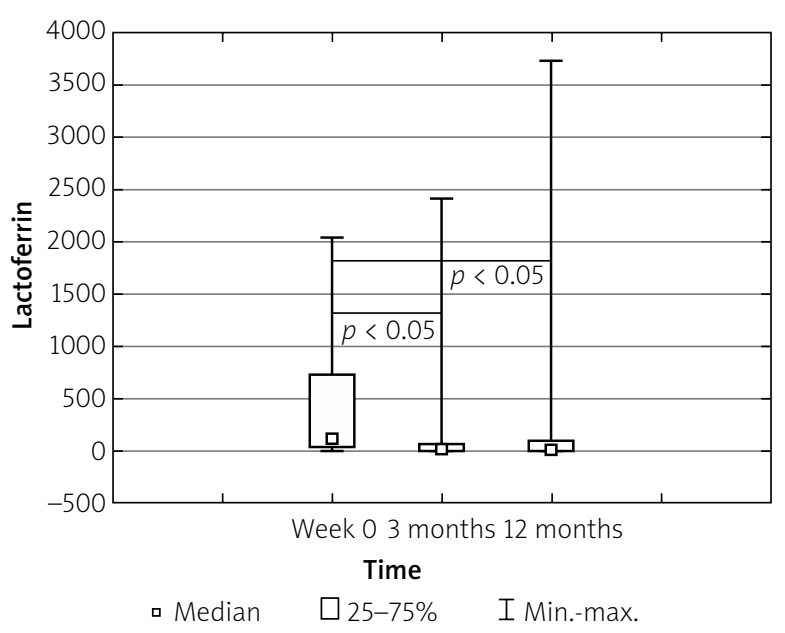

Figure 2. Median faecal lactoferrin concentrations before anti-TNF treatment, after 3 months, and after 12 months of the therapy

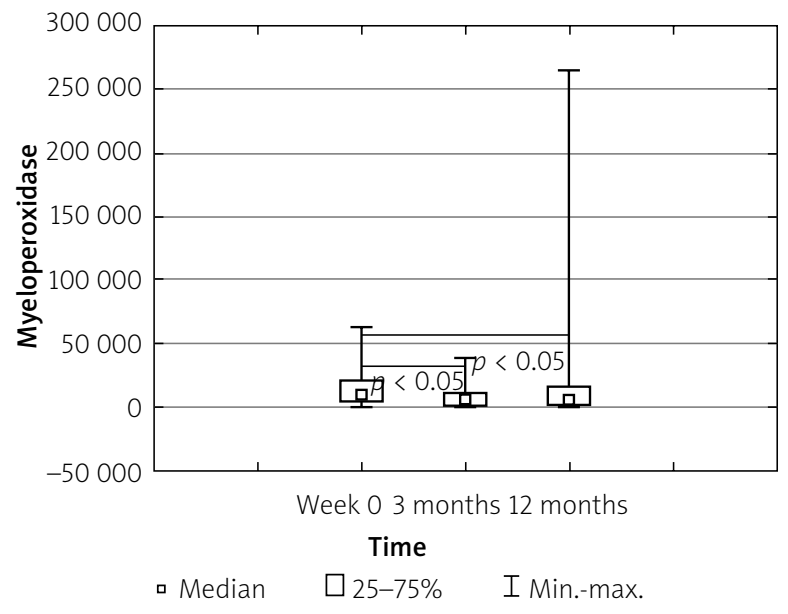

Figure 3. Median faecal myeloperoxidase concentrations before anti-TNF treatment, after 3 months, and after 12 months of the therapy

\section{Discussion}

In patients treated with biological agents, it is very important to evaluate the effectiveness of the administered therapy. This assessment should take into account both the clinical response and endoscopic response to treatment. Based on numerous studies it has been proven that the remission of mucosal changes is a necessary element in obtaining a permanent treatment response $[12,17]$. In order to evaluate the clinical activity of CD, CDAl is the most commonly used scale, both in every day practice, as well as during clinical trials and scientific studies. It is worth mentioning that CDAI applies to all the studies that evaluate the effectiveness of biological treatment. The clinical response is extremely important; 
Table III. Correlation analysis between assessed faecal marker concentrations, Crohn's Disease Activity Index (CDAI), and Simple Endoscopic Score for Crohn's Disease (SES-CD) before starting anti-TNF treatment (week 0)

\begin{tabular}{lccc} 
Variable & Calprotectin & Lactoferrin & $\begin{array}{c}\text { Myeloperoxi- } \\
\text { dase }\end{array}$ \\
\hline CDAI & $p<0.0001$ & $p<0.05$ & NS \\
& $r=0.431$ & $r=0.304$ & $r=0.244$ \\
\hline SES-CD & $p<0.05$ & $p<0.05$ & NS \\
& $r=0.420$ & $r=0.484$ & $r=0.258$
\end{tabular}

Table IV. Correlation analysis between assessed faecal marker concentrations, Crohn's Disease Activity Index (CDAI), and Simple Endoscopic Score for Crohn's Disease (SES-CD) after 3 months of anti-TNF treatment (week 12)

\begin{tabular}{lccc} 
Variable & Calprotectin & Lactoferrin & $\begin{array}{c}\text { Myeloperoxi- } \\
\text { dase }\end{array}$ \\
\hline CDAI & $p<0.05$ & $p<0.05$ & NS \\
& $r=0.310$ & $r=0.300$ & $r=0.254$ \\
\hline SES-CD & $p<0.05$ & $p<0.05$ & $p<0.05$ \\
& $r=0.420$ & $r=0.395$ & $r=0.379$
\end{tabular}

Table V. Correlation analysis between assessed faecal marker concentrations, Crohn's Disease Activity Index (CDAI), and Simple Endoscopic Score for Crohn's Disease (SES-CD) after 12 months of anti-TNF treatment (week 56)

\begin{tabular}{lccc} 
Variable & Calprotectin & Lactoferrin & $\begin{array}{c}\text { Myeloperoxi- } \\
\text { dase }\end{array}$ \\
\hline CDAI & $p<0.0001$ & $p<0.05$ & $p<0.05$ \\
& $r=0.461$ & $r=0.391$ & $r=0.399$ \\
\hline SES-CD & $p<0.0001$ & $p<0.05$ & $p<0.05$ \\
& $r=0.562$ & $r=0.556$ & $r=0.384$
\end{tabular}

however, it does not necessarily reflect the endoscopic changes $[2,3,5]$. This is mostly due to the fact that the clinical activity index consists of a lot of subjective measures, and on the other hand a high endoscopic activity does not always fully reflect the clinical picture. In the clinical practice we often observe patients with high CDAl index but the endoscopic activity is very low, and vice versa. Therefore, in order to correctly evaluate the endoscopic activity, it is necessary to perform a colonoscopy. As previously mentioned it is an invasive and expensive examination. With better access to biological treatment nowadays, strict monitoring of treatment response using endoscopic examinations is nearly impossible. That is why it is necessary to search for cheaper and less invasive methods. Based on the recent literature it has been proposed that faecal biomarkers could be an accurate measure of endoscopic activity [18, 19].
Most of these studies concern patients before the treatment initiation. There are not many studies evaluating the usefulness of faecal biomarkers in monitoring of the response to anti-TNF- $\alpha$ treatment. As is commonly known, biological treatment consists of medications that significantly affect $\mathrm{MH}$. They are products that also significantly affect immunological processes in the gastrointestinal tract. It is also important to note that patients who undergo biological treatment very often have high endoscopic activity index and a more severe disease course. That is why, in order to correctly use the faecal markers to evaluate the biological treatment response, it is necessary to perform more studies on this group of patients. In our opinion transferring the results from other groups of patients is not adequate. In our study we attempted to evaluate the usefulness of selected faecal markers in the assessment of response to biological treatment during 1 year of administration.

The results of our study confirm the usefulness of both calprotectin and lactoferrin in the evaluation of endoscopic activity of the disease before the administration of biological treatment. We obtained the correlations between these measures. The concentration of these faecal markers correlated with the clinical activity of the disease, which in our opinion is noteworthy. It validates the fact that high values of calprotectin and lactoferrin can be a good early diagnostic tool during disease exacerbations. Our results are consistent with other studies [18, 19].

In literature, there have not been many studies that focus on the use of faecal markers in the biological treatment. Based on the published data we cannot make any assumptions about the usefulness of faecal markers in monitoring the biological treatment response. Sipponen et al. showed that the concentration of calprotectin and lactoferrin in stool decreases in patients who responded well to treatment. It was not observed in the group of patients who did not respond well to treatment. These studies included patients before the administration of a biological treatment and after the induction time, which is after 3 months of treatment. The authors of this study concluded that the faecal markers could be useful in monitoring the biological treatment response [20]. Different conclusions were drawn by Nogueira et al. Their studied group consisted of 17 patients who underwent biological treatment. They measured the concentrations of calprotectin and lactoferrin in stool before the treatment administration, after 8 weeks of induction period, and at week 32 of treatment. The researchers showed that before the administration of biological treatment faecal markers can be an accurate measure of endoscopic and histological remission, but during the course of the disease they do 
not correlate as precisely with the endoscopic activity of the disease. The authors concluded that the biomarkers are useful in the long-term evaluation of biological treatment [21]. Our results are different to these observations, but consistent with data from Sipponen et al. In our study, during the course of biological treatment the concentration of both calprotectin and lactoferrin decreased, which still correlated with the endoscopic and clinical activity of the disease. Based on our study we can draw the conclusion that lactoferrin and calprotectin can be accurate measures of endoscopic remission in patients treated with biological treatment. It could be very beneficial to monitor the concentration individually in each patient. It is especially important in patients after induction of remission with biological treatment. In this group of patients a complete remission of the mucosal changes is very rare. That is why it is very important to observe a decrease in the concentration of both calprotectin and lactoferrin in comparison to the baseline levels. On the other hand, after 12 months of therapy when a full $\mathrm{MH}$ is obtained, the concentration of biomarkers should further decrease. Both calprotectin and lactoferrin can be very useful measures in monitoring the treatment response because of their high stability at room temperature. Obtaining a sample is very easy and noninvasive, and the fact that the temperature does not affect their concentration is an additional advantage.

In the literature there are no studies concerning the usefulness of myeloperoxidase in the evaluation of biological treatment response. In our study we found a correlation between the concentration of myeloperoxidase in stool and the endoscopic activity after the induction period and after a year of therapy. It is important to note that we did not find such a correlation in patients before the administration of treatment. It is also interesting that the correlation between clinical activity and the concentration of myeloperoxidase in stool was only present after 12 months of therapy. As we mentioned above, this is a pioneering study in this topic. Based on the results it is still too early to draw any conclusions about the usefulness of myeloperoxidase in monitoring biological treatment response. The lack of correlation between the concentration of myeloperoxidase and the endoscopic and clinical activity before the administration of treatment is a significant limitation. Therefore, there is still a question whether observing the changes in concentrations of this faecal marker in individual patients in comparison to initial concentrations will be an accurate reflection of endoscopic changes and the response to treatment. It is necessary to perform more studies in this topic.

Throughout the period of 16 years of biological treatment administration there have been numerous observations evaluating the effectiveness of the response to treatment. The term "deep remission" was created. It consists of both the biochemical and endoscopic response. The combination of two types of response seems to be very important. It is associated with the fact that in some patients, despite the lack of endoscopic changes, there can still be some microscopic changes. The elevated levels of biomarkers in stool can be a reflection of this. Only normalisation of all the components represents "deep remission" [22, 23]. It is not easy to accomplish it. It seems that in everyday practice it is more important to monitor the concentrations of faecal markers individually in selected patients and not to normalise them.

\section{Conclusions}

In our study we presented that faecal calprotectin and lactoferrin are surrogate markers for endoscopic and clinical activity of CD. They are useful in monitoring the biological treatment response. It cannot be concluded whether the concentration of myeloperoxidase in stool is useful in the evaluation of treatment response. More research needs to be conducted in this field.

\section{Acknowledgments}

This study was funded by a grant from the Ministry of Science and Higher Education in Poland (NN402 481 637).

\section{Conflict of interest}

The authors declare no conflict of interest.

\section{References}

1. Nikolaus S, Schreiber S. Diagnostics of inflammatory bowel disease. Gastroenterology 2007; 133: 1670-89.

2. Best WR, Becktel JM, Singleton JW, et al. Development of a Crohn's disease activity index. Gastroenterology 1976; 70 : 439-44.

3. Harvey RF, Bradshaw JM. A simple index of Crohn's disease activity. Lancet 1980; 1: 514.

4. Daperno M, D'Haens G, Van Assche G, et al. Development and validation of a new, simplified endoscopic activity score for Crohn's disease: the SES-CD. Gastrointest Endosc 2004; 60: 505-12.

5. Sostegni R, Daperno M, Scaglione N, et al. Crohn's disease: monitoring disease activity. Aliment Pharmacol Ther 2003; 17 Suppl 2: 11-7.

6. Walsh A, Palmer R, Travis S. Mucosal healing as a target of therapy for colonic inflammatory bowel disease and methods to score disease activity. Gastrointest Endosc Clin N Am 2014; 24: 367-78

7. Costa F, Mumolo MG, Bellini M, et al. Role of faecal calprotectin as non-invasive marker of intestinal inflammation. Dig Liver Dis 2003; 35: 642-7. 
8. Iskandar HN, Ciorba MA. Biomarkers in inflammatory bowel disease: current practices and recent advances. Transl Res 2012; 159: 313-25.

9. Jones J, Loftus EV Jr, Panaccione R, et al. Relationships between disease activity and serum and fecal biomarkers in patients with Crohn's disease. Clin Gastroenterol Hepatol 2008; 6: 1218-24.

10. Gaya DR, Lyon TD, Duncan A, et al. Faecal calprotectin in the assessment of Crohn's disease activity. QJM 2005; 98: 435-41.

11. D'Haens G, Ferrante M, Vermeire S, et al. Fecal calprotectin is a surrogate marker for endoscopic lesions in inflammatory bowel disease. Inflamm Bowel Dis 2012; 18: 221-4.

12. Ford AC, Sandborn WJ, Khan KJ, et al. Efficacy of biological therapies in inflammatory bowel disease: systematic review and meta-analysis. Am J Gastroenterol 2011; 106: 644-59.

13. Kondera-Anasz Z, Marek Z, Mielczarek-Palacz A, et al. Kalprotektyna - budowa i funkcje. Pol Arch Med Wewn 2006; 3: 248-53.

14. Kerkhoff C, Vogel T, Nacken W, et al. Zinc binding reverses the calcium-induced arachidonic acid-binding capacity of the S100A8/A9 protein complex. FEBS Lett 1999; 460: 134-8.

15. Baveye S, Elass E, Mazurier J, et al. Lactoferrin: a multifunctional glycoprotein involved in the modulation of the inflammatory process. Clin Chem Lab Med 1999; 37: 281-6.

16. Winterbourn CC, Vissers MC, Kettle AJ. Myeloperoxidase. Curr Opin Hematol 2000; 7: 53-8.

17. Pineton de Chambrun G, Peyrin-Biroulet L, Lémann M, et al. Clinical implications of mucosal healing for the management of IBD. Nat Rev Gastroenterol Hepatol 2010; 7: 15-29.

18. Sipponen T, Savilahti E, Kolho KL, et al. Crohn's disease activity assessed by fecal calprotectin and lactoferrin: correlation with Crohn's disease activity index and endoscopic findings. Inflamm Bowel Dis 2008; 14: 40-6.

19. Schoepfer AM, Beglinger C, Straumann A, et al. Ulcerative colitis: correlation of the Rachmilewitz endoscopic activity index with fecal calprotectin, clinical activity, C-reactive protein, and blood leukocytes. Inflamm Bowel Dis 2009; 15: 1851-8.

20. Sipponen T, Savilahti E, Kärkkäinen P, et al. Fecal calprotectin, lactoferrin, and endoscopic disease activity in monitoring anti-TNF-alpha therapy for Crohn's disease. Inflamm Bowel Dis 2008; 14: 1392-8.

21. Nogueira IM, Miszputen SJ, Ambrogini Jr O, et al. Assessment of the response of patients with Crohn's disease to biological therapy using new non-invasive markers: lactoferrin and calprotectin. Arq Gastroenterol 2013; 50: 130-7.

22. Molander P, Farkkila M, Salminen K, et al. Outcome after discontinuation of TNF-alpha-blocking therapy in patients with inflammatory bowel disease in deep remission. Inflamm Bowel Dis 2014; 20: 1021-8.

23. Louis E, Mary JY, Vernier-Massouille G; Groupe D'etudes Therapeutiques Des Affections Inflammatoires Digestives. Maintenance of remission among patients with Crohn's disease on antimetabolite therapy after infliximab therapy is stopped. Gastroenterology 2012; 142: 63-70.

Received: 15.05 .2015

Accepted: 10.07 .2015 